\title{
AN UNUSUAL LOWER NECK MASS
}

Uma Patnaik ${ }^{1}$, Gurmeet Singh ${ }^{2}$

HOW TO CITE THIS ARTICLE:

Uma Patnaik, Gurmeet Singh. "An unusual lower neck mass". Journal of Evolution of Medical and Dental Sciences 2013; Vol2, Issue 40, October 07; Page: 7632-7636.

ABSTRACT: Mucous retention cysts are fairly common in the minor salivary glands. Submandibular gland retention cysts are relatively rare with very few cases reported in literature presenting as an upper neck mass. We present an unusual case of submandibular gland retention cyst presenting as a lower lateral neck mass. Clinical examination did not reveal origin from submandibular salivary gland. It was only during surgery that the cyst was found to be in continuation with the submandibular gland. Extensive search of literature did not reveal submandibular mucus retention cyst presenting as a mass in the lower neck.

KEY WORDS: mucus retention cyst, submandibular salivary gland, lateral neck mass

INTRODUCTION: Mucous retention cysts of the minor salivary glands are commonly encountered in clinical practice. However, they are a rare occurrence in major salivary glands. Submandibular mucocele is fairly uncommon with very few cases reported. ${ }^{1-4}$ Plunging ranula communicate with the neck through a defect in the mylohyoid muscle, usually arising from the sublingual salivary gland. These may present as a swelling in the submental or submandibular triangle. Submandibular mucoceles and mucous retention cysts presenting in the neck have been reported very rarely and the few reported cases have presented as submandibular mass. Our case presented as a lower lateral neck mass which to our knowledge have not been reported. Clinical examination did not reveal origin from submandibular salivary gland. It was only during surgery that the cyst was found to be in continuation with the submandibular gland.

CASE REPORT: 47 yr old patient, chronic beedi smoker presented with swelling in the left side of the neck of one month duration. The onset of the swelling was insidious in onset, initially size of a pea with gradual increase in size to that of a lemon. There was no history of pain, sudden increase in size, dysphagia, voice change, similar swelling in the neck or rest of the body, fever, night sweats or features of hyper or hypothyroidism.

Clinically, general and systemic examination was normal. Local examination of the neck revealed soft, cystic, oval $4 \times 3 \mathrm{~cm}$ sized swelling in the muscular triangle on the left side extending from hyoid to thyroid cartilage which was fluctuant, mobile on deglutition, non pulsatile and carotid was felt separately from the swelling (Figure 1). The left submandibular gland was clinically normal and there was no stone palpable in the Wharton's duct. Indirect laryngoscopy and examination of the ear, nose and throat was normal. The USG was suggestive of a cystic swelling. FNAC showed mucous retention cyst of salivary gland. On MRI well defined cystic lesion seen on the left side of the neck extending from hyoid to thyroid cartilage, superficial to strap muscles and thyroid cartilage (Figure 2). The patient was planned for surgery. Under general anesthesia, horizontal skin crease incision was taken over most prominent part of the cyst. Sub platysmal flaps were elevated and dense adhesions were found between the cyst and strap muscles. The cyst was found to be in continuation with the left submandibular gland. It was excised in continuation with the gland 
(Figure 3), with preservation of the marginal mandibular, hypoglossal and lingual nerves. The submandibular duct was found to be thickened and fibrosed. After securing hemostasis, drain was placed and incision sutured in layers. Post operative recovery was uneventful with preserved functions of marginal mandibular and hypoglossal nerves. Histopathology report was suggestive of mucous retention cyst with non specific chronic sialoadenitis of the submandibular gland. The patient was followed up for one year and no recurrence was reported.

DISCUSSION: A submandibular gland retention cyst is a possibility in a lower lateral neck mass as reported by us. The various other differential diagnosis of a lateral neck mass is listed at Table1.

Mucus retention cysts commonly arise from the minor salivary glands of the lip, floor of mouth or buccal mucosa. Submandibular gland retention cysts are extremely uncommon. Anastassov et $\mathrm{al}^{4}$ ha ve mentioned only 5 cases in a review of English literature and 2 cases treated by them. Back and Fahny have reported submandibular duct cyst resembling a laryngocele in the submandibular region. ${ }^{5}$ Submandibular mucocele is fairly uncommon with very few cases reported. Felstead and Patel have reported a submandibular mucocele presenting in the submandibular region. ${ }^{6}$ Mucus retention cyst with an origin from the submandibular gland presenting as a mass in the lower neck has not been reported. This rarity is probably due to the fact that the gland is protected by its anatomical location.

Plunging ranulas are usually associated with the sublingual salivary glands in the floor of the mouth. An origin from ectopic salivary glands located on the inferior surface of mylohyoid has been hypothesised.7 Oral ranulas are due to mucus extravasation superior to mylohyoid muscles. Plunging ranula which presents as a swelling in the submental or submandibular triangle is due to mucus escape into the neck through the fascial planes below the mylohyoid muscle. The radiological finding of "Tail sign" characteristic of the plunging ranula.

In our patient the submandibular gland was clinically normal and no stone or mucopus discharge was present in the Wharton's duct. A probable origin from the submandibular gland was noted on the MRI and it was only during surgery that a continuation of the swelling with the submandibular gland was confirmed.

The excision of the cyst alone would have a possibility of salivary fistula as a postoperative complication, hence we decided to excise the submandibular gland along with the cyst. Prior consent of the patient had been taken for this. Also, as the patient had risk factors like chronic beedi smoking and old age, the possibility of salivary gland malignancy presenting as a retention cyst was there. Hence, the gland was also sent for histopathological examination.

The mucocele, the ranula, and the cervical, or plunging, ranula are clinical terms for a pseudocyst that is associated with mucus extravasation into the surrounding soft tissues . Mucus retention cysts are true cysts lined by epithelium. The various aetiologies for retention cyst include idiopathic, traumatic, obstructive, infective, developmental and neoplastic. The possibility of a neoplastic aetiology was there in our patient due to his age and associated risk factor of beedi smoking.

The management options include both conservative and surgical measures. Surgical treatment is the preferred option, the principle of treatment being excision of the cyst along with the affected salivary gland to avoid recurrences. Conservative measures mentioned are repeated aspiration and drainage of cyst cavity. ${ }^{8}$ Injection of sclerosing agents like OK 432 is in the experimental stages. ${ }^{9}$ Also, $\mathrm{CO}_{2}$ Laser and low dose radiation therapy have been used for 
treatment. ${ }^{10}$ In our patient complete excision of the cyst along with the affected submandibular gland was done in continuation.

Mucus retention cyst of the submandibular salivary gland can present as a lower lateral neck mass as reported by us. To our knowledge, this presentation has not been reported. However, the possibility of a mucus retention cyst of the submandibular salivary gland must be kept in mind in the differential diagnosis of a lower lateral neck mass. We recommend complete excision of the cyst along with the affected salivary gland.

\section{REFERENCES}

1. Surkin M, Remsen K, Lawson W, Som P, Biller HF. A mucocele of the submandibular gland. Arch Otolaryngol 1985;111:623-5

2. Okumura K, Inui M, Nakase M, Nakamura S, Hiramota K, Tagawa T. A case of submandibular gland mucocoele. Journal of Clinical Paediatric Dentistry 2008;31:207-9

3. Shingo K, Takashi S, Masashi H, Itaru N, Tooru K. Submandibular gland mucocoele of the extravasation type: report of a case Hosp Dent Oral- Maxillofac Surg 2003;15:71-4

4. Anastassov GE, Haiavy J, Solodnik P, Lee H, Lumerman H. Submandibular gland mucocoele: diagnosis and management. Oral Surg Oral Med Oral Pathol Oral Radiol Endod 2000;89(2):159-63

5. Back GW, Fahmy F, Hosni A. Submandibular salivary duct cyst mimicking an external laryngocoele. Journal of Laryngology and Otology 2000;114(4):305-7

6. Felstead AM, Patel PM, Revington PJ. Submandibular gland mucocoele presenting as a lateral neck swelling, JCSR 2012:6-8

7. Ichimura K, Ohta Y, Tayama N. Surgical management of the plunging ranula: a review of seven cases. J Larngol Otol 1996;110(6):554-6

8. Bonet FB, Homs EV, Tornil AM. Submaxillary gland mucocoele: presentation of a case. Med Oral Patol Oral Cir Bucal 2005;10:180-4

9. Woo JS, Hwang SJ, LeeHM. Recurrent plunging ranula treated with OK-432. European Archives of Oto-Rhino-Laryngology 2003; 260, 226-8

10. Mintz S, Barak S, Horowitz I. Carbon dioxide laser excision and vaporization of nonplunging ranulas: a comparison of two treatment protocols. Journal of Oral and Maxillofacial Surgery $1994 ; 52: 370-2$

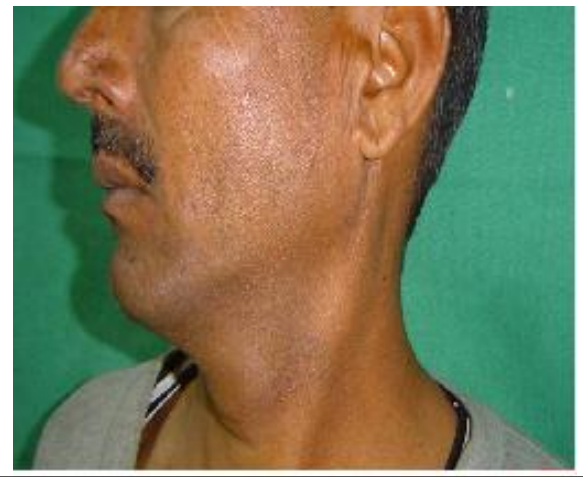

Figure 1: Cystic swelling located in the muscular triangle (left) 


\section{CASE REPORT}
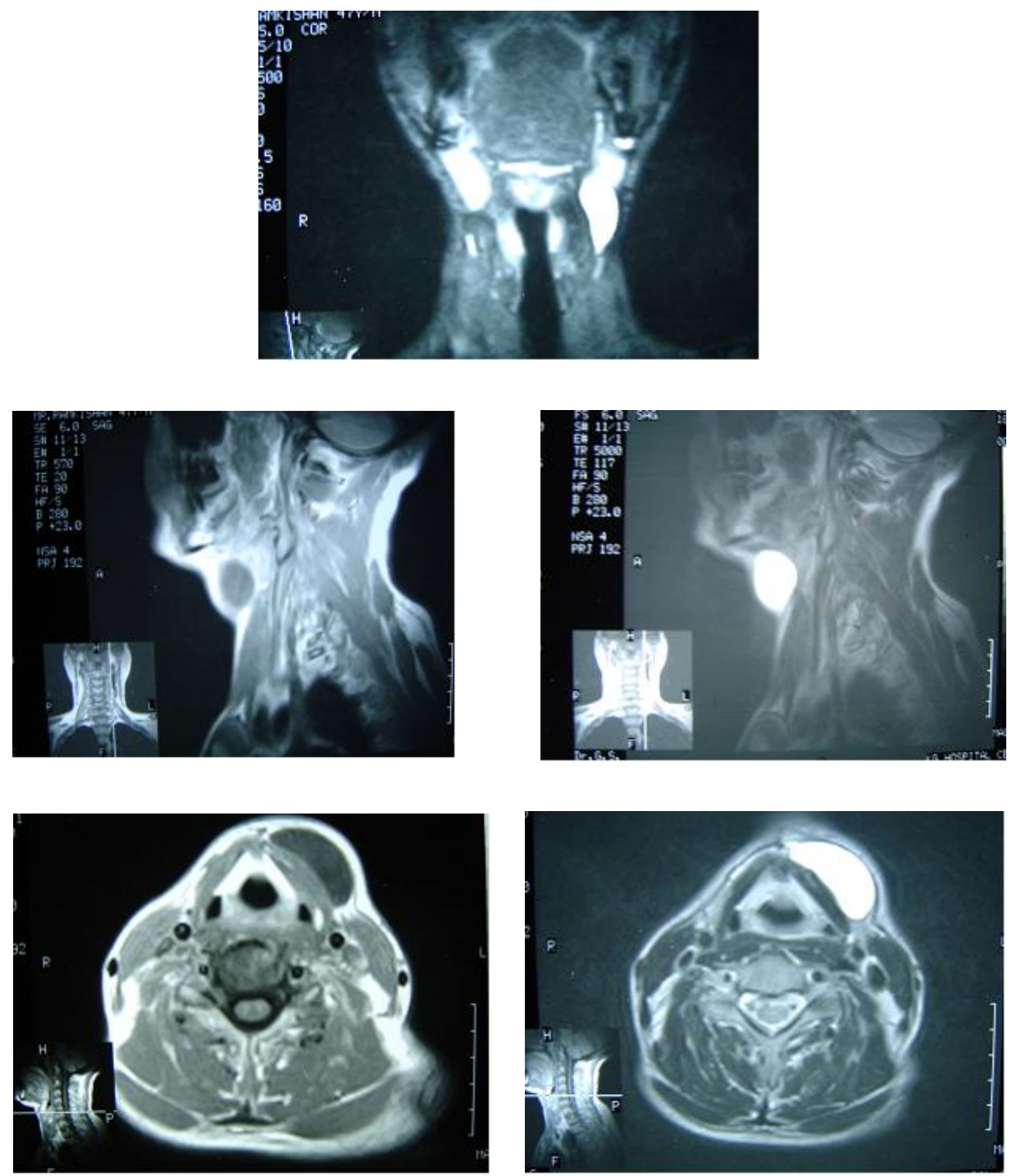

Figure 2: MRI findings: well defined cystic lesion just below strap muscles, hypointense on $\mathrm{T} 1$, hyperintense on $\mathrm{T} 2$ inferior to left submandibular gland extending from hyoid to thyroid cartilage.

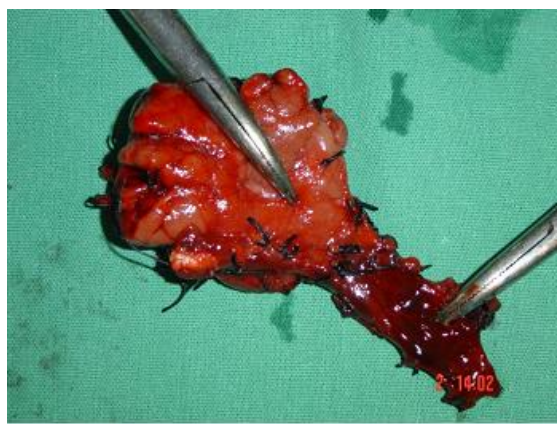

Figure 3: Specimen showing cyst along with submandibular salivary gland excised in continuity. 


\section{AUTHORS:}

1. Uma Patnaik

2. Gurmeet Singh

\section{PARTICULARS OF CONTRIBUTORS:}

1. Assistant Professor, Department of ENT, Military Hospital, Hisar, Tamil Nadu, India.

2. Assistant Professor, Department of Surgery, Military Hospital, Hisar, Tamil Nadu, India.

\section{NAME ADDRESS EMAIL ID OF THE CORRESPONDING AUTHOR:}

Dr. Uma Patnaik,

Military Hospital, Hisar,

C/O, 56, APO.

Email-umi75pat@gmail.com

Date of Submission: 02/09/2013.

Date of Peer Review: 03/09/2013.

Date of Acceptance: 13/09/2013.

Date of Publishing: 01/10/2013 